\title{
Tangeretin enhances radiosensitivity and inhibits the radiation-induced epithelial-mesenchymal transition of gastric cancer cells
}

\author{
XUKUI ZHANG ${ }^{1}$, LUMING ZHENG ${ }^{1}$, YINGGANG SUN ${ }^{1}$, TIANXIAO WANG ${ }^{2}$ and BAOCHENG WANG ${ }^{3}$ \\ ${ }^{1}$ Department of General Surgery, PLA Jinan General Hospital, Jinan, Shandong 250031; ${ }^{2}$ Medical School, \\ Shangdong University, Jinan, Shandong 250012; ${ }^{3}$ Department of Oncology, \\ PLA Jinan General Hospital, Jinan, Shandong 250031, P.R. China \\ Received January 8, 2015; Accepted April 3, 2015
}

DOI: 10.3892/or.2015.3982

\begin{abstract}
Irradiation has been reported to increase radioresistance and epithelial-mesenchymal transition (EMT) in gastric cancer (GC) cells. The Notch pathway is critically implicated in cancer EMT and radioresistance. In the present study, we investigated the use of a Notch-1 inhibiting compound as a novel therapeutic candidate to regulate radiation-induced EMT in GC cells. According to previous screening, tangeretin, a polymethoxylated flavonoid from citrus fruits was selected as a Notch-1 inhibitor. Tangeretin enhanced the radiosensitivity of GC cells as demonstrated by MTT and colony formation assays. Tangeretin also attenuated radiation-induced EMT, invasion and migration in GC cells, accompanied by a decrease in Notch-1, Jagged1/2, Hey-1 and Hes-1 expressions. Tangeretin triggered the upregulation of miR-410, a tumor-suppressive microRNA. Furthermore, re-expression of miR-410 prevented radiation-induced EMT and cell invasion. An in vivo tumor xenograft model confirmed the antimetastasis effect of tangeretin as we observed in vitro. In nude mice, tumor size was considerably diminished by radiation plus tangeretin co-treatment. Tangeretin almost completely inhibited lung metastasis induced by irradiation. Tangeretin may be a novel antimetastatic agent for radiotherapy.
\end{abstract}

\section{Introduction}

Gastric cancer (GC) remains a severe public health problem worldwide. In China, GC is often diagnosed at an advanced clinical stage, characteristic of obvious lymphatic tumor

Correspondence to: Professor Baocheng Wang, Department of Oncology, PLA Jinan General Hospital, 25 Shifan Road, Jinan 250031, P.R. China

E-mail: wangbaochengpla@126.com

Key words: radiation, epithelial-mesenchymal transition, invasion, Notch-1, tangeretin, miR-410 dissemination (1). Radiotherapy is the main modality for unresectable GC (http://www.nccn.org/index.asp) (2).

However, radiation is also a double-edged sword; it not only kills tumor cells, yet also promotes radioresistance and induces distant metastases, and destroys normal tissues (3). The intricate process of metastasis includes a series of divergent steps. Epithelial-mesenchymal transition (EMT) is one of the main programs. The EMT process leads to acquisition of mesenchymal characteristics, including motility, invasiveness, chemoresistance and radioresistance (4). Uncovering the relationship between irradiation and EMT is essential. E-cadherin, which facilitates and ensures the continuous adhesive epithelium, is a biomarker of the EMT process (5). The loss of E-cadherin leads to the lack of stable intercellular junctions; therefore cell dispersion is accelerated due to the decrease in cellular adhesive forces. Jung et al reported that the morphology of cells changes after irradiation and cells appeared similar to fibroblasts corresponding to a mesenchymal phenotype (6).

The Notch axis is a key participant in EMT. Notch signaling is a conserved family of transmembrane receptors that determines cell fate (7). It includes 4 Notch family members (Notch-1-4), 5 Notch ligands, $3 \delta$-like ligands (Dll1/3/4) and 2 serrate-like ligands (Jagged1/2) (8). Notch is dysregulated in various types of cancers, accompanied by poor clinical outcomes (9). Notch signaling can be activated under radiation and there is accumulating evidence confirming the use of Notch inhibitors as promising radiosensitizers in cancer treatment. Notch has also been proven to mediate radioresistance in nasopharyngeal carcinoma and glioma cells (10-12).

MicroRNAs (miRNAs) are a class of small non-coding RNAs of 21-23 nucleotides that control specific mRNA translation or induce mRNA degradation (13). Dysregulated miRNA expression is well correlated with various malignancies. The deficiency in members of the miR-200 family (miR-200s), miR-124 and let-7, are observed in certain types of cancers, and are regarded as tumor suppressors (14). In contrast, various overexpressed miRNAs, such as miR-373 and $\mathrm{miR}-21$, are regarded as oncogenes, involved in cell proliferation and metastasis (15). In GC tissues, the expression levels of miR-221, miR-222, miR 21 and miR-103 were found to be 
significantly higher than levels in normal samples. However, the expression of miR-143 and miR-195 in cancer samples was significantly lower than levels in normal tissues (16). Moreover, miR-200c deficiency promoted cancer metastasis, EMT and aggressiveness (17).

As a polymethoxylated flavonoid abundant in citrus fruits, tangeretin (5,6,7,8,4'-pentamethoxyflavone) has been reported to display apoptotic (18), antimetastatic (19) and antioxidant properties in various cancer models. It inhibited the proliferation and induced G1 phase arrest of MCF-7 cells after a 4-day treatment (20). Tangeretin induced the apoptosis of human leukemia cells (18) and enhanced the cytotoxic effect of doxorubicin on breast cancer cells (21). Importantly, tangeretin possessed little toxicity as there was no significant weight loss and no obvious change in the gross behavior of rats (22). In our previous studies (unpublished data), we screened for effective radiosensitizers of GC cells. Recently, we observed the potential radiosensitizing effects of tangeretin on GC cells, which led us to explore the mechanism involved.

\section{Materials and methods}

Materials. Tangeretin (98\%) was purchased from the National Institute for the Control of Pharmaceutical and Biological Products (Beijing, China). Tangeretin was dissolved in dimethylsulfoxide (DMSO) for all in vitro experiments. DMSO, RPMI-1640 medium, fetal calf serum (FBS), Tween-20, sodium dodecyl sulfate (SDS), phenylmethylsulphonyl fluoride (PMSF), trypsin and carbonylcyanide p-trifluoromethoxyphenylhydrazone (FCCP) were purchased from Sigma Chemical Co. (St. Louis, MO, USA). Notch-1 siRNA and miR-410 mimics were obtained from Dharmacon (Chicago, IL, USA). The Notch-1 cDNA plasmid was purchased from OriBioGene Biological Inc. (Shanghai, China). Deionized water was purified by a Milli-Q water purification system (Millipore, Milford, MA, USA). All other reagents were of analytical grade and obtained from Nanjing Chemical Reagent Co. (Nanjing, China).

Cell lines and cell viability. GC cell lines (MGC80-3, AGS, SGC7901 and MKN45) and normal gastric mucosa GES-1 cells were purchased from the American Type Culture Collection (ATCC; Rockville, MD, USA) and were cultured in RPMI-1640 supplemented with 10\% FBS. Cell viability was determined using the 3-(4,5-dimethylthiazol-2-yl)-2,5-diphenyltetrazolium bromide (MTT) assay. Radiosensitivity was examined by colony formation assay. Cells were exposed to increasing doses of irradiation (2, 4, 6 and $8 \mathrm{~Gy})$ and tangeretin for $24 \mathrm{~h}$. Irradiation was produced with a $4-\mathrm{MeV}$ electron beam accelerator (Elekta, Sweden). After being washed with fresh medium, cells were allowed to grow for 14 days to form colonies, and cells were then fixed with $4 \%$ formaldehyde (Sigma). Fixed cells were then stained with $0.5 \%$ crystal violet (Sigma) for $15 \mathrm{~min}$, and the colonies were counted. D0 values were calculated by a multitarget-single hit model.

In vivo study protocol. Athymic male nude mice weighing $\sim 20 \mathrm{~g}$ (SLARC Laboratory Animal Center, Shanghai, China) were used and maintained under standard pathogen-free conditions. SGC7901 cells were harvested and injected subcutaneously into the nude mice. After the onset of tumor development, tangeretin [30 mg/kg dissolved in $1 \mathrm{ml}$ of phosphate-buffered saline (PBS) containing 0.1\% DMSO/nude mice] was administered intraperitoneally for 3 weeks. After the mice were anesthetized, the tumors were exposed to radiation with 2 Gy 5 times a week for 3 weeks. After 3 weeks of administration, all mice were sacrificed and the tumors were subjected to further analysis. The numbers of lung metastatic lesions were counted under a microscope (Zeiss Axio Observer A1; Zeiss, Germany).

Western blot analysis. Cells were extracted in extraction buffer. Equal amounts of protein extracts were separated on $10 \%$ polyacrylamide gels and electrophoretically transferred onto polyvinylidene difluoride membranes (Invitrogen, Carlsband, CA, USA) with a semi-dry blot system. After blocking, the membranes were incubated with each primary antibody (Nanjing Bioworld Biotech Co., Nanjing, China) and then with IgG.

Cell invasion assays. After co-treatment with tangeretin (10 and $30 \mu \mathrm{M}$ ) and radiation ( $8 \mathrm{~Gy}$ ) for $24 \mathrm{~h}$, the cells were then placed in the upper chamber of a Transwell coated with BD Matrigel ${ }^{\mathrm{TM}}$. Medium with $10 \%$ FBS was placed in the lower chamber. After $18 \mathrm{~h}$, the cells that had migrated to the lower surface were photographed and quantified in 5 fields at a magnification of x100 under a microscope (Zeiss Axio Observer A1).

Wound healing assay. Cells were seeded in 24-well plates and grown until reaching confluency. In each well, a scratch was made with a 1-ml pipette tip. The cells were washed thrice with PBS to remove detached cells. Then the cells were treated with irradiation (8 Gy) and tangeretin (10 and $30 \mu \mathrm{M})$, and images of the wound area were captured at 0 and $18 \mathrm{~h}$ under a microscope (Zeiss Axio Observer A1).

Immunofluorescence microscopy. After exposure to irradiation ( $8 \mathrm{~Gy}$ ) for $24 \mathrm{~h}$, immunofluorescence assay was performed to examine EMT biomarkers in the SGC7901 cells. After fixation and permeabilization, E-cadherin and $\mathrm{N}$-cadherin were measured following staining with anti-E-cadherin and anti-N-cadherin antibodies. Nuclei were counterstained with 4',6-diamidine-2'-phenylindole dihydrochloride (DAPI) (Sigma Chemical Co.). The stained cells were observed with a fluorescence inverted microscope (Zeiss Axio Observer A1).

miRNA microarray analysis. Total RNA was harvested using miRNeasy Mini kit (Qiagen, Copenhagen, Denmark) according to the manufacturer's instructions $(23,24)$. The profiles of samples were generated using Agilent Human miRNA Microarray V3 (Agilent Technologies, Inc., Santa Clara, CA, USA). The scanned images were imported into GenePix Pro 6.0 software (Axon Instruments, Molecular Devices Corp., Foster City, CA, USA) for data extraction. Real-time PCR was carried out to confirm the miRNA expression profiling with U6 as the internal reference gene.

Statistical analysis. Data are expressed as the mean \pm SD from 3 independent experiments. Group results were analyzed using 

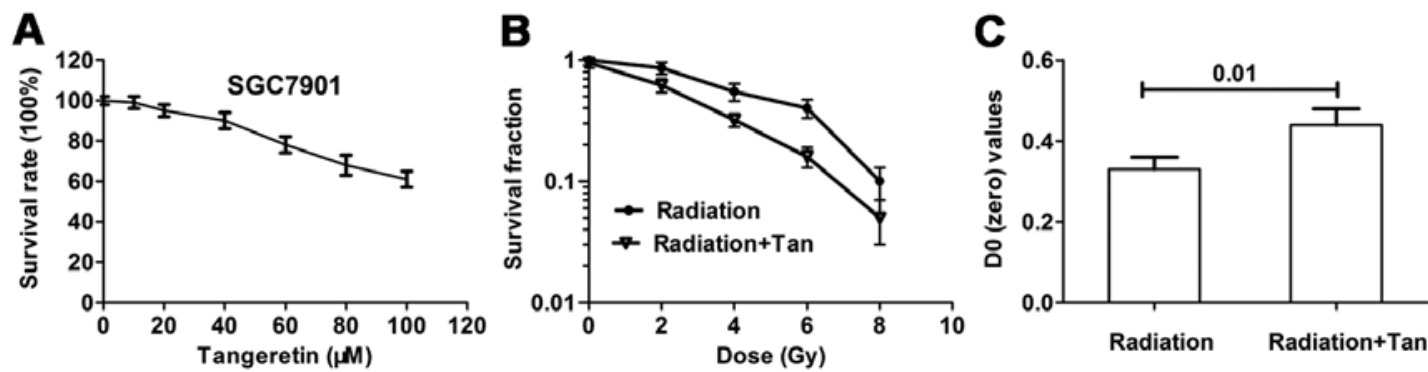

Figure 1. Tangeretin increases the radiosensitivity of SGC7901 cells. (A) MTT assay. Survival rates after incubation with tangeretin (Tan) (0-100 $\mu \mathrm{M})$ for $24 \mathrm{~h}$. (B) Clonogenic survival assay. Cells were exposed to radiation (2, 4, 6 and $8 \mathrm{~Gy})$ with or without a subtoxic concentration of tangeretin ( $30 \mu \mathrm{M})$ for $24 \mathrm{~h}$. After being washed with fresh medium, the cells were allowed to grow for 14 days to form colonies. (C) D0 value.
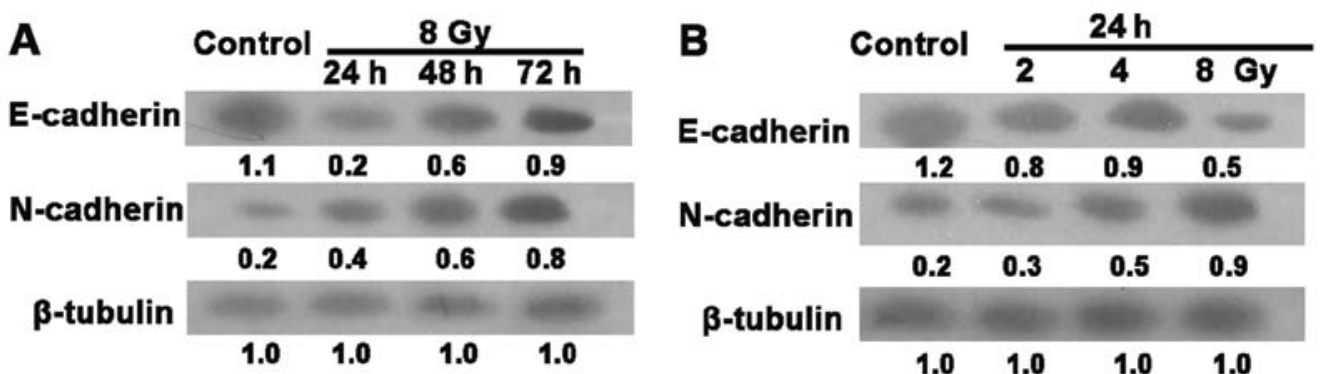

C

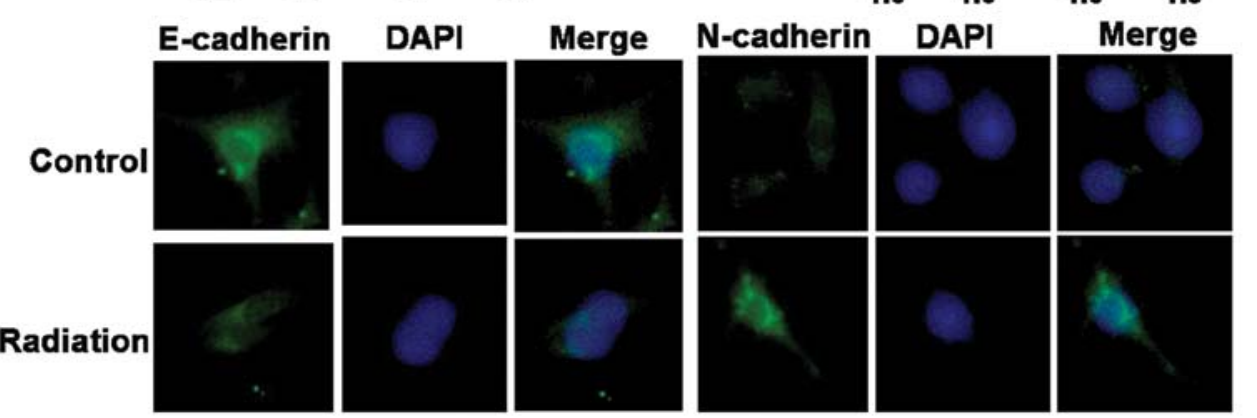

Figure 2. Irradiation modulates EMT markers in SGC7901 cells in a time- and dose-dependent manner. (A and B) SGC7901 cells were irradiated with increasing doses of irradiation (2, 4 and $8 \mathrm{~Gy}$ ) and were harvested at 24, 48 and $72 \mathrm{~h}$ post-irradiation. Biomarkers for EMT were analyzed by western blot analysis. $\beta$-tubulin was used as a loading control. (C) After irradiation (8 Gy) for $24 \mathrm{~h}$, SGC7901 cells were harvested, and an immunofluorescence assay was carried out to determine EMT biomarker expression. EMT, epithelial-mesenchymal transition.

one-way analysis of variance (ANOVA). Two groups were analyzed by the Student's t-test. P-value $<0.05$ was considered to indicate a statistically significant result.

\section{Results}

Tangeretin decreases cell viability and increase radiosensitivity in the SGC7901 cells. Cytotoxicity was detected by MTT assay. Cells were treated with tangeretin $(0-100 \mu \mathrm{M})$ for $24 \mathrm{~h}$. Tangeretin caused a cytotoxicity effect on the SGC7901 cells in a dose-dependent manner. Tangeretin did not affect cell viability until reaching concentrations of $30 \mu \mathrm{M}$ (Fig. 1A). The non-cytotoxic concentrations used for the present study were identified, to ensure that the radiosensitizing effect of tangeretin was not caused by a direct cytotoxic effect.

A colony forming assay was carried out to explore whether tangeretin increased radiosensitivity in the SGC7901 cells. Cell survival curves measured by clonogenic survival assay are illustrated in Fig. 1B. Tangeretin considerably increased radiation-induced cell clonogenic death. D0 of the tangeretin plus radiation group was significantly increased $(\mathrm{P}=0.01)$, compared with the radiation alone group (Fig. 1C).

Radiation induces EMT in the gastric cancer cells. The loss of the epithelial phenotype marker, E-cadherin, is obvious in the EMT process. Features of the mesenchymal phenotype, such as augmented formation of pseudopodia and the appearance of spindle-shaped cells, were observed at $24 \mathrm{~h}$ post-irradiation (data not shown). To determine whether this alteration correlates with EMT, we examined EMT-specific markers by immunofluorescence assay and western blotting. As shown in Fig. 2A and B, radiation induced a decrease in E-cadherin and promoted the expression of the mesenchymal marker N-cadherin in the SGC7901 cells. Similar results were observed in the immunofluorescence assay (Fig. 2C). These data suggest that radiation induced EMT in the human GC SGC7901 cells.

Tangeretin reduces radiation-induced EMT and invasion and migration in the SGC7901 cells. Tangeretin suppressed 


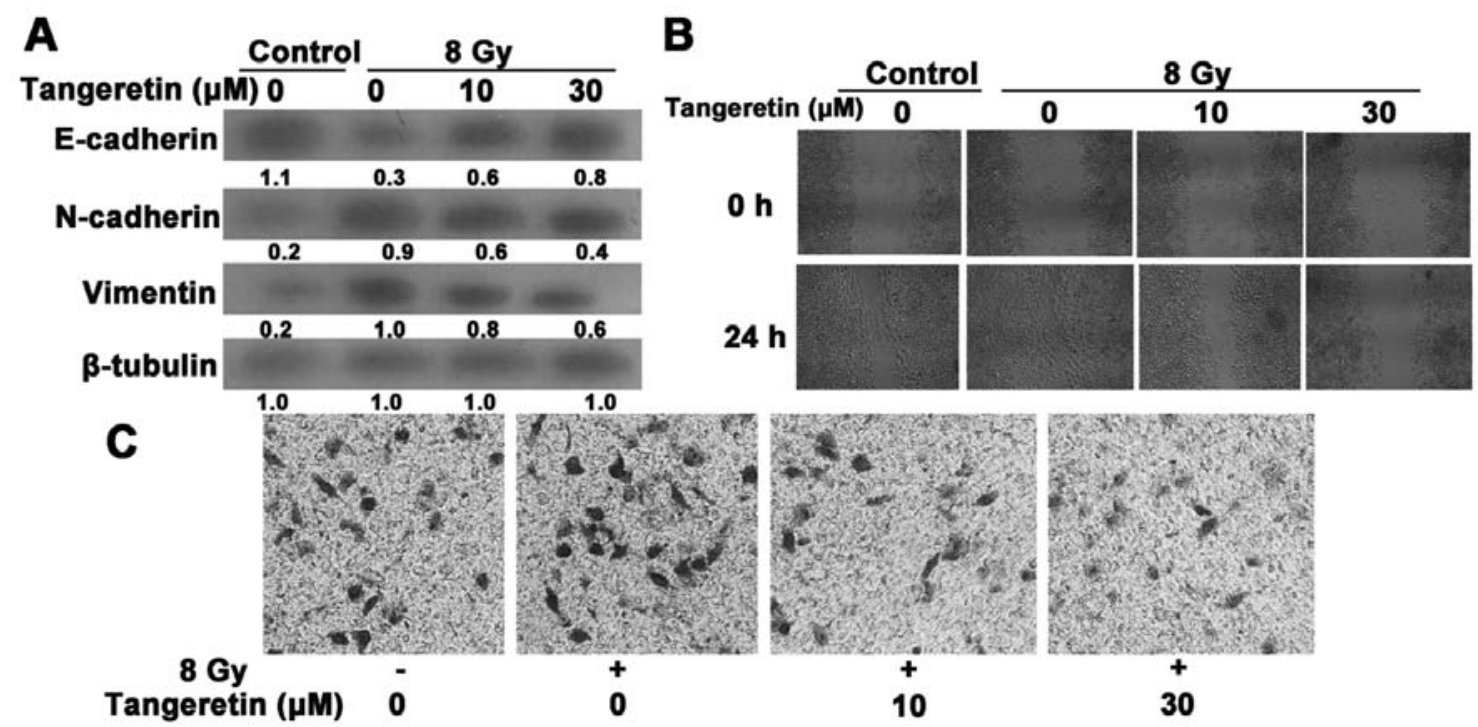

Figure 3. Tangeretin suppressed EMT, invasion and migration induced by radiation. Cells were co-treated with tangeretin $(10$ and $30 \mu \mathrm{M})$ and radiation $(8 \mathrm{~Gy})$ for $24 \mathrm{~h}$. (A) Biomarkers for EMT were evaluated by western blot analysis. (B) Wound closure assays. Cells were co-treated with radiation and tangeretin (10 and $30 \mu \mathrm{M}$ ) after wound scratches were made. Images were captured at 0 and $18 \mathrm{~h}$ after wounding. (C) Invasion assays. After co-treatment with radiation and tangeretin for $24 \mathrm{~h}$, the invasive ability was measured by Transwells. The number of invaded SGC7901 cells in 5 fields at a magnification of x100 was counted under a microscope. EMT, epithelial-mesenchymal transition.
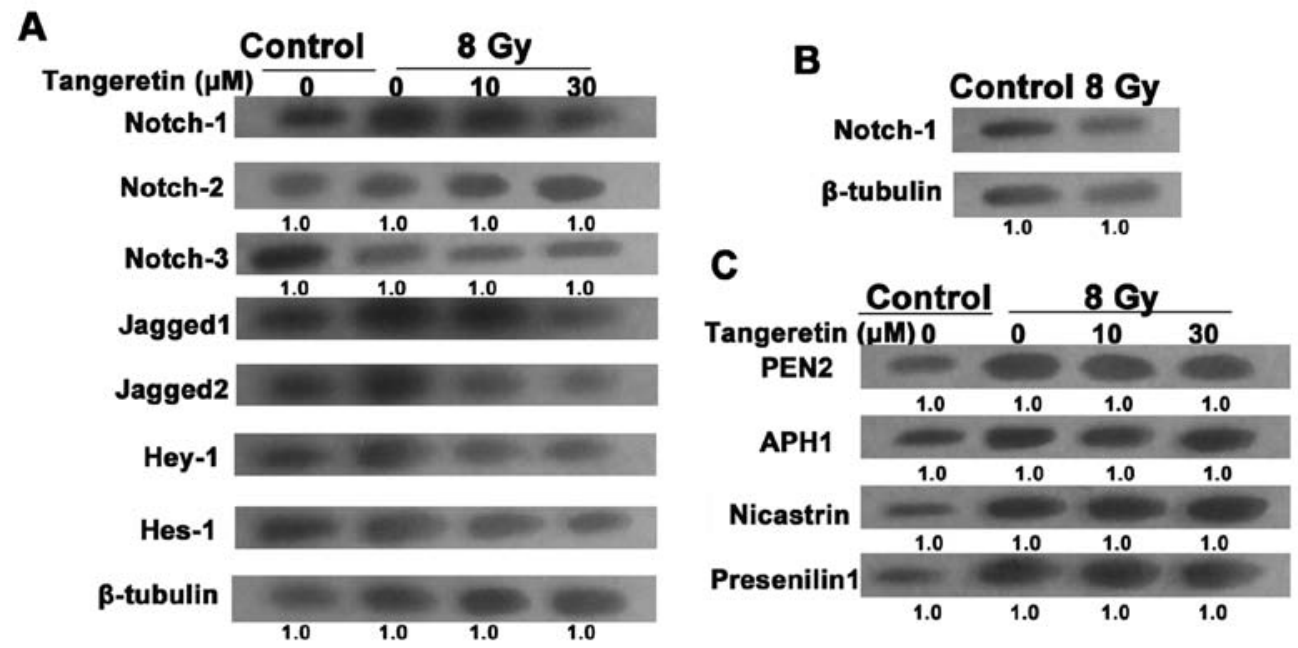

Figure 4. Tangeretin inhibits the Notch-1 pathway in irradiated SGC7901 cells. Cells were co-treated with radiation and tangeretin for $24 \mathrm{~h}$, and the cells were harvested $24 \mathrm{~h}$ post-radiation. (A) Expression levels of Notch-1, Notch-2, Notch-3, Hes-1, Hey-1, Jagged1 and Jagged2 were detected by western blotting. (B) Notch-1 expression in the GES-1 cells. (C) $\gamma$-secretase complex proteins.

radiation-induced EMT, as revealed by a reduced reduction in expression of vimentin and $\mathrm{N}$-cadherin and an increase in expression of E-cadherin (Fig. 3A). The EMT process is also accompanied with enhanced cell invasion and migration. We further evaluated the invasive and migratory properties of SGC7901 cells via Matrigel Transwell and wound-healing assays. Radiation accelerated the rate of wound closure (Fig. 3B). Likewise, the acquisition of increased invasive ability induced by irradiation was prohibited by tangeretin treatment (Fig. 3C). These data indicate that tangeretin diminished the radiation-induced responses in SGC7901 cells.

Tangeretin inhibits the Notch-1 pathway in the irradiated SGC7901 cells. Evidence has proven the essential role of the Notch-1 pathway in the EMT process (25). To uncover the molecular mechanism responsible for radiation-induced EMT, the expression of the Notch pathway in irradiated cells was examined. After treatment with tangeretin for $24 \mathrm{~h}$, the upregulation of Notch-1, Jagged1/2, Hes-1 and Hey-1 expression levels in the irradiated cells was almost blocked. However, radiation or/and tangeretin co-incubation failed to influence the expression of Notch-2/3 (Fig. 4A). Moreover, western blot assays showed that there was no significant change in Notch-1 expression in the gastric mucosa GES-1 cells after exposure to irradiation (Fig. 4B).

After binding to Jagged or $\delta$ ligands, the Notch receptor is activated and goes through a succession of cleavages. The final cleavage depends on the $\gamma$-secretase protease complex, which 


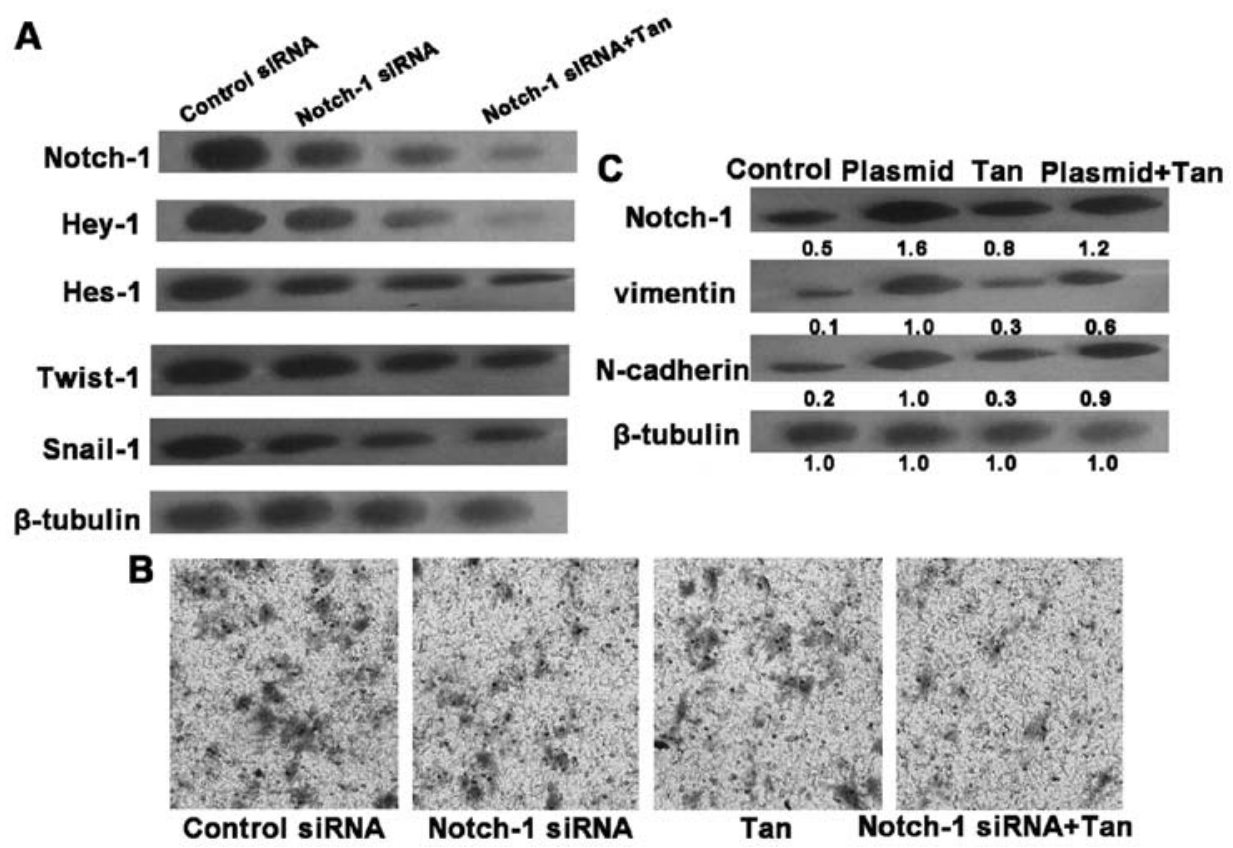

Figure 5. Knockdown of Notch-1 alleviates EMT in SGC7901 cells. (A) Cells were transfected with a control or a Notch-1-specific siRNA. After 24 h post-transfection, cells were irradiated at $8 \mathrm{~Gy}$ with or without tangeretin (Tan) $(10 \mu \mathrm{M})$ co-treatment and cell lysates were collected to measure EMT-related protein levels. (B) Invasion assays. After $24 \mathrm{~h}$ post-transfection, cells were irradiated at $8 \mathrm{~Gy}$ with or without tangeretin $(10 \mu \mathrm{M})$ co-treatment, and the invasive ability was measured by Transwells. (C) Overexpression of Notch-1. Cells were transfected with empty vector or plasmid of Notch-1. After 24 h, cells were irradiated at $8 \mathrm{~Gy}$ with or without tangeretin $(30 \mu \mathrm{M})$ co-treatment. EMT biomarkers were determined by western blot assay. EMT, epithelial-mesenchymal transition.
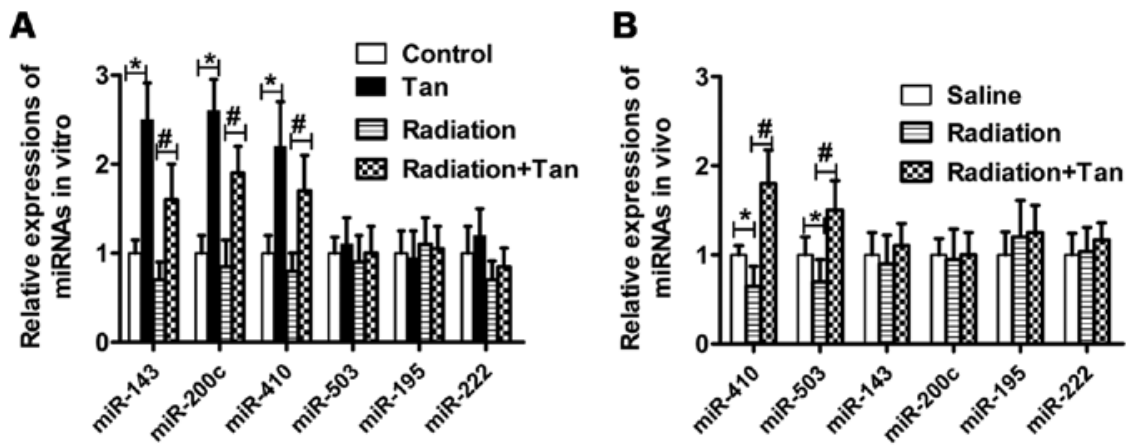

Figure 6. Tangeretin elevates miR-410 expression both in vitro and in vivo. (A) miRNA levels in vitro. SGC7901 cells were exposed to radiation and tangeretin (Tan) $(30 \mu \mathrm{M})$ for 24 or $48 \mathrm{~h}$. ${ }^{*} \mathrm{P}<0.05$, compared with medium control; ${ }^{~} \mathrm{P}<0.05$, compared with radiation alone. (B) miRNA levels in vivo. After the onset of tumors, tangeretin $(30 \mathrm{mg} / \mathrm{kg} \mathrm{BW})$ was administered intraperitoneally for another 3 weeks, and then the tumor tissues were removed to determine miRNA levels $(\mathrm{n}=5)$. ${ }^{*} \mathrm{P}<0.05$, compared with the saline group; ${ }^{*} \mathrm{P}<0.05$, compared with the radiation alone group.

contains 4 catalytic subunits such as PEN2, presenilin 1, APH1 and nicastrin (26), causing the translocation of Notch into the nucleus (27). We then tested the effect of tangeretin on the activation of Notch-1 signaling. Tangeretin did not influence the protein level of the 4 catalytic subunits, suggesting that tangeretin only suppressed the expression rather than the activation of Notch-1 (Fig. 4C).

Knockdown of Notch-1 alleviates EMT in SGC7901 cells. To further demonstrate the essential role of Notch-1, the Notch-1 gene was knocked down by siRNA. Notch-1 siRNA transfection downregulated the protein levels of Notch-1, Hey-1, Hes-1, Snail1 and Twist1 in the irradiated SGC7901 cells, accompanied with a decline in invasive ability. After $24 \mathrm{~h}$ post-transfection, tangeretin treatment inhibited both Notch-1 activity and cell invasion to a more significant degree in the irradiated SGC7901 cells than tangeretin treatment alone (Fig. 5A and B). We then explored the relationship between tangeretin and Notch-1 with the overexpression plasmid of Notch-1. Overexpression of Notch-1 overrode the inhibitory effect of tangeretin on EMT (Fig. 5C). Accordingly, we hypothesized that tangeretin prevented EMT probably via suppressing Notch-1 expression.

Tangeretin elevates miR-410 expression both in vitro and in vivo. Radiation regulates miRNA expression, and in turn affects tumor progression. As demonstrated here, in SGC7901 cells exposed to tangeretin, enhanced expression of miR-143, miR-200c and miR-410 was noted when compared to the control cells. Moreover, the relative change in miR-410 expression was greater in the tangeretin plus irradiation group, than the radiation alone group (Fig. 6A). In addition, tangeretin 

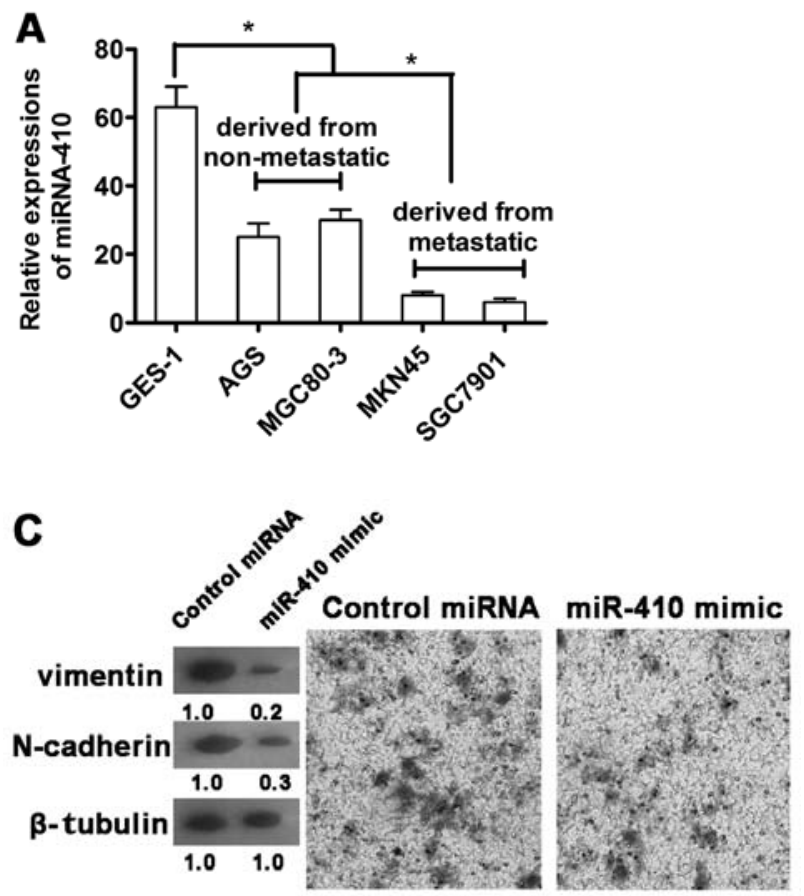
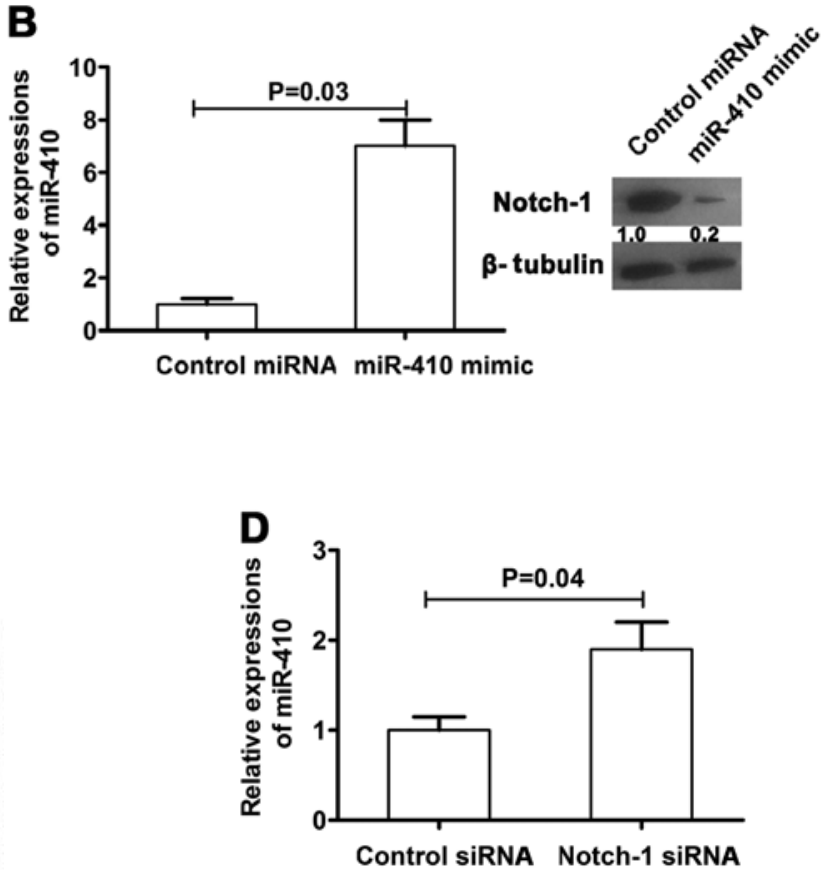

Figure 7. Overexpression of miR-410 inhibits EMT. (A) qRT-PCR analysis of miR-410 expression in 4 GC cell lines and GES-1 cells. U6 was used as the internal reference gene. (B) qRT-PCR analysis of miR-410 expression and western blot analysis of Notch-1 expression in irradiated-SGC7901 cells transfected with miR-410 mimics. U6 was used as the internal reference gene. (C) Western blotting and Transwell analysis in the irradiated-SGC7901 cells transfected with miR-410 mimics. (D) qRT-PCR analysis of miR-410 expression after knockdown of Notch-1 in the irradiated SGC7901 cells. EMT, epithelial-mesenchymal transition.
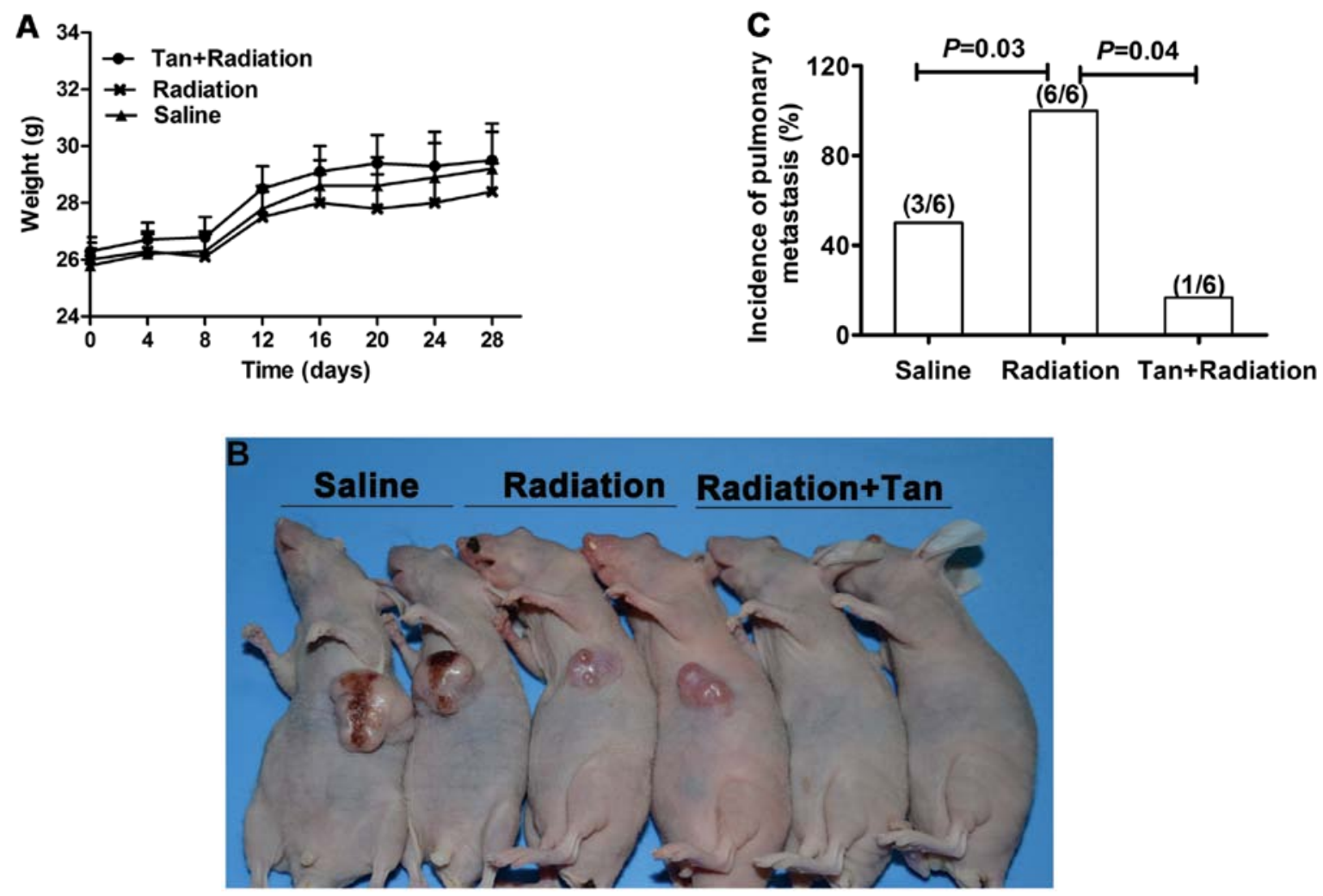

Figure 8. Radioenhancement efficacy of tangeretin in vivo. (A) Changes in body weight. (B) Tumor volumes. (C) Pulmonary metastasis.

promoted the expression of miR-410 and miR-503 in tumor tissue lysates from nude mice (Fig. 6B). These results demon- strated that tangeretin elevated miR-410 expression both in vitro and in vivo. 
miR-410 expression is reduced in gastric camcer cells. We then compared miR-410 expression between GC cell lines (MGC80-3, AGS, SGC7901 and MKN45) and the normal gastric mucosa GES-1 cells. miR-410 expression was reduced in the GC cell lines compared to the GES-1 cells. MGC80-3 and AGS cell lines were derived from non-metastatic tissues, and SGC7901 and MKN45 cell lines were from metastatic tissues. Expression of miR-410 was higher in the MGC80-3 and AGS cells than those derived from metastatic tissues $(\mathrm{P}<0.05$, Fig. 7A).

miR-410 plays a role in the biologic function of tangeretin in EMT. Since miRNAs are crucial modulators of EMT and Notch-1 signaling, we further investigated the effect of miR-410 on the EMT of GC cells subjected to radiation. Enhanced miR-410 expression and weakened Notch-1 expression were observed in the irradiated-SGC7901 cells after miR-410 mimics were transfected into the cells (Fig. 7B). Overexpression of miR-410 also reduced EMT-specific marker expression and invasion in the irradiated cells, indicating that miR-410 is closely associated with EMT (Fig. 7C). In addition, miR-410 expression was upregulated after the knockdown of Notch-1 by siRNA in the irradiated SGC7901 cells, and these functions were similar to the effect by tangeretin (Fig. 7D). In summary, miR-410 plays an essential role in the biologic function of tangeretin in EMT.

Effects of tangeretin in vivo. Body weight partly reflects a healthy condition. A decrease in body weight was observed in the group exposed to radiation for 3 weeks. Tangeretin considerably alleviated radiation-induced weight loss in nude mice after 3 weeks of administration. During the period of administration, mice in the tangeretin + radiation group were in better physical condition and exhibited higher weight gain, compared with the radiation group (Fig. 8A).

Following treatment with tangeretin + radiation for 3 weeks, the tumor sizes were smallest among all the groups at the observation endpoint (Fig. 8B). The incidence of pulmonary metastasis in the radiotherapy and control groups was 100 and $50 \%$ (6/6 vs. 3/6, P<0.05; Fig. 8C). Compared with the radiation group, the tangeretin + radiation group had considerably attenuated lung metastases, with metastatic rates of $16.67 \%$ (1/6 vs. $6 / 6, \mathrm{P}<0.05)$.

\section{Discussion}

The National Comprehensive Cancer Network guidelines recommend radiotherapy as a standard treatment for gastric cancer (GC) patients with a high-risk of recurrence (28). However, radiotherapy has shown the tendency to induce metastasis. Moreover, increased irradiation has toxic effects on the skin and other normal tissues (29). Identification of radiosensitizers that maximize radiotherapeutic efficacy and minimize toxicity has attracted worldwide attention. In the present study, we showed that tangeretin enhanced the radiosensitivity of SGC7901 cells and suppressed irradiationinduced epithelial-mesenchymal transition (EMT) and metastasis both in vitro and in vivo, probably via inhibition of Notch-1 signaling transduction and the 'switching on' of miR-410. This opens perspectives for the development of novel radiosensitizers in GC therapy. Moreover, the absorption and pharmacokinetics of tangeretin have been studied both in vivo and in vitro. In hamsters administered $1 \%$ tangeretin flavone for 35 days, total tangeretin and tangeretin metabolites reached levels equivalent to $21 \mu \mathrm{M}$ intact tangeretin in serum and 16-67 $\mu \mathrm{M}$ in liver (30). In rats intraperitoneally administered tangeretin $(50 \mathrm{mg} / \mathrm{kg})$, the concentration of tangeretin in serum reached a peak of $12.1 \mu \mathrm{M}$ at $0.5 \mathrm{~h}$ (31). In the present study, the concentrations of tangeretin $(10$ and $30 \mu \mathrm{M})$ used in vitro were comparable to the levels achievable in vivo. Nevertheless, the detailed metabolic features of tangeretin in nude mice bearing tumor xenografts warrant more profound study.

Mounting evidence indicates that radiation is one of the inducers of EMT, andEMT directly induces radioresistance (32). Irregular EMT activation in the stomach is closely related with gastric carcinogenesis (33). EMT activation endows gastric epithelial cells with augmented characteristics of mesenchymal cells and decreases their epithelial features. Radiation-induced metastasis is also closely related to a mesenchymal phenotype. E-cadherin, an epithelial biomarker, is a member of a large superfamily of cell-cell adhesion molecules (34). During EMT, cadherin changes from E-cadherin to N-cadherin which is expressed in mesenchymal cells. Thus, decreased E-cadherin and increased $\mathrm{N}$-cadherin are noted in the EMT process, and both can be identified as EMT biomarkers. Tangeretin has been found to inhibit invasion of cancer cells in an E-cadherindependent manner (34). Tangeretin also reduced the number of metastatic nodules in mice bearing B16F10 cell xenografts (35). In the present study, a decrease in E-cadherin as well as a simultaneous increase in $\mathrm{N}$-cadherin and vimentin were observed after exposure to radiation, indicating that the epithelial cells acquired a mesenchymal-like morphology. Tangeretin successfully inhibited E-cadherin expression, invasion and migration induced by irradiation.

The Notch-1 pathway plays an important role in EMT progression (25). Blocking Notch-1 signaling by Hey-1 or Jagged1 knockdown mitigated EMT (36). As an oncogene in various solid malignancies, Notch-1 is expressed in most GC cell lines and normal gastric mucosa. The $\gamma$-secretase inhibitor DAPT (one of the GSIs) successfully inhibited the EMT and metastasis of GC cells. There is crosstalk between the EMT transcription factors and Notch such as Slug and Snail (37). Notably, Du et al showed that increased expression of Notch-1 is associated with non-cardia location, positive lymphovascular invasion, diffuse type, tumor size $>5 \mathrm{~cm}$ and distal metastasis in GC cancer patients (38); Notch-1 may be regarded as a poor prognostic predictor in GC. Consistently, we found that Notch-1 was expressed in both SGC7901 cells and normal mucosa GES-1 cells, but a higher level was observed in SGC7901 cells than in GES-1 cells after exposure to irradiation, suggesting that Notch-1 is activated under irradiation. Tangeretin exhibited potent Notch-1 inhibiting capacity. However, tangeretin only suppressed the expression of Notch-1, not the activation of Notch-1. Furthermore, the overexpression of Notch-1 overrode the inhibitory effect of tangeretin on EMT. Consequently, Notch-1 may be a target of tangeretin in the inhibitory effect on EMT.

Recently, a mount of evidence strongly supports the multifactorial role of miRNAs in critical cellular processes. In particular, miRNAs may function as tumor-suppressor genes 
or oncogenes. In the present study, we compared the expression of 6 miRNAs (miR-410, miR-143, miR-195, miR-222, miR-200c and miR-503) between GC cell lines/tissues and normal gastric cells/tissue. Studies indicate that miR-143 and 200c play an important role in blocking cancer progression $(13,39)$. Our data revealed that tangeretin promoted miR-143 and miR-200c expression only in vitro, without a significant effect in vivo. The miR-503 level has been reported to be considerably reduced in GC tissues compared to normal mucosa tissues in 76 patients who experienced gastric surgery between 2012 and 2013 (16). miR-503 expression levels also negatively correlated with metastases in patients. The present study demonstrated that tangeretin only promoted miR-503 in tumor tissue from nude mice. Zhang el al reported that upregulation of miR-222 induced the malignant phenotype of SGC7901 cells, whereas knockdown of miR-222 reversed this phenotype (40). In the present study, tangeretin displayed no effect on miR-222 expression in the SGC7901 cells. In the present study, in SGC7901 cells, as well as in tumor tissues, combined treatment of tangeretin and irradiation upregulated the expression of miR-410. Gattolliat et al showed that the miR-410 level was considerably related with disease-free survival of the non-amplified neuroblastoma (41). Shen et al suggested that miR-410 acts as a tumor suppressor by targeting the MDM2 gene and inhibiting GC cell proliferation, migration and invasion (42). Similarly, we found that miR-410 expression was lower in the SGC7901 cells than that in the normal gastric mucosa cells. Moreover, the miR-410 level was higher in cells derived from non-metastatic tissues than those derived from metastatic tissues.

We further investigated the correlation between miR-410 and Notch-1. Overexpression of miR-410 significantly weakened Notch-1 expression and inhibited the EMT process in irradiated GC cells. In addition, Notch-1 siRNA led to the enhancement of miR-410 expression in the irradiated SGC7901 cells. Tangeretin treatment also promoted miR-410 expression in the irradiated SGC7901 cells.

Taken together, we conclude that both Notch-1 and miR-410 are key effectors in the biologic function of tangeretin. Inactivation of Notch-1 signaling leads to the reversal of EMT, along with less invasive characteristics. Tangeretin plus radiation has noteworthy potential as an effective anti-GC strategy.

\section{References}

1. Bandres E, Bitarte N, Arias F, Agorreta J, Fortes P, Agirre X, Zarate R, Diaz-Gonzalez JA, Ramirez N, Sola JJ, et al: microRNA-451 regulates macrophage migration inhibitory factor production and proliferation of gastrointestinal cancer cells. Clin Cancer Res 15: 2281-2290, 2009.

2. Hartgrink HH, Jansen EP, van Grieken NC and van de Velde CJ: Gastric cancer. Lancet 374: 477-490, 2009.

3. Liu W, Huang YJ, Liu C, Yang YY, Liu H, Cui JG, Cheng Y, Gao F, Cai JM and Li BL: Inhibition of TBK1 attenuates radiation-induced epithelial-mesenchymal transition of A549 human lung cancer cells via activation of GSK-3 $\beta$ and repression of ZEB1. Lab Invest 94: 362-370, 2014.

4. Cui FB, Liu Q, Li RT, Shen J, Wu PY, Yu LX, Hu WJ, Wu FL, Jiang CP, Yue GF, et al: Enhancement of radiotherapy efficacy by miR-200c-loaded gelatinase-stimuli PEG-Pep-PCL nanoparticles in gastric cancer cells. Int J Nanomed 9: 2345-2358, 2014.

5. Moncharmont C, Levy A, Guy JB, Falk AT, Guilbert M, Trone JC, Alphonse G, Gilormini M, Ardail D, Toillon RA, et al: Radiation-enhanced cell migration/invasion process: A review. Crit Rev Oncol Hematol 92: 133-142, 2014.
6. Jung JW, Hwang SY, Hwang JS, Oh ES, Park S and Han IO: Ionising radiation induces changes associated with epithelialmesenchymal transdifferentiation and increased cell motility of A549 lung epithelial cells. Eur J Cancer 43: 1214-1224, 2007.

7. Artavanis-Tsakonas S, Rand MD and Lake RJ: Notch signaling: Cell fate control and signal integration in development. Science 284: 770-776, 1999.

8. D'Souza B, Miyamoto A and Weinmaster G: The many facets of Notch ligands. Oncogene 27: 5148-5167, 2008.

9. Espinoza I and Miele L: Notch inhibitors for cancer treatment. Pharmacol Ther 139: 95-110, 2013.

10. Wang J, Wakeman TP, Lathia JD, Hjelmeland AB, Wang XF, White RR, Rich JN and Sullenger BA: Notch promotes radioresistance of glioma stem cells. Stem Cells 28: 17-28, 2010.

11. Phillips TM, McBride WH and Pajonk F: The response of $\mathrm{CD} 24^{- \text {/low }} / \mathrm{CD} 44^{+}$breast cancer-initiating cells to radiation. J Natl Cancer Inst 98: 1777-1785, 2006.

12. Yu S, Zhang R, Liu F, Hu H, Yu S and Wang H: Down-regulation of Notch signaling by a $\gamma$-secretase inhibitor enhances the radiosensitivity of nasopharyngeal carcinoma cells. Oncol Rep 26: 1323-1328, 2011.

13. Wu XL, Cheng B, Li PY, Huang HJ, Zhao Q, Dan ZL, Tian DA and Zhang P: MicroRNA-143 suppresses gastric cancer cell growth and induces apoptosis by targeting COX-2. World $\mathrm{J}$ Gastroenterol 19: 7758-7765, 2013.

14. Liang YJ, Wang QY, Zhou CX, Yin QQ, He M, Yu XT, Cao DX, Chen GQ, He JR and Zhao Q: MiR-124 targets Slug to regulate epithelial-mesenchymal transition and metastasis of breast cancer. Carcinogenesis 34: 713-722, 2013.

15. Ye X, Jiang F, Li Y, Mu J, Si L, Wang X, Ning S and Li Z: Glabridin attenuates the migratory and invasive capacity of breast cancer cells by activating microRNA-200c. Cancer Sci 105: 875-882, 2014.

16. Guo B, Li J, Liu L, Hou N, Chang D, Zhao L, Li Z, Song T and Huang C: Dysregulation of miRNAs and their potential as biomarkers for the diagnosis of gastric cancer. Biomed Rep 1: 907-912, 2013.

17. Cortez MA, Valdecanas D, Zhang X, Zhan Y, Bhardwaj V, Calin GA, Komaki R, Giri DK, Quini CC, Wolfe T, et al: Therapeutic delivery of miR-200c enhances radiosensitivity in lung cancer. Mol Ther 22: 1494-1503, 2014.

18. Hirano T, Abe K, Gotoh M and Oka K: Citrus flavone tangeretin inhibits leukaemic HL-60 cell growth partially through induction of apoptosis with less cytotoxicity on normal lymphocytes. Br J Cancer 72: 1380-1388, 1995.

19. Seo J, Lee HS, Ryoo S, Seo JH, Min BS and Lee JH: Tangeretin, a citrus flavonoid, inhibits PGDF-BB-induced proliferation and migration of aortic smooth muscle cells by blocking AKT activation. Eur J Pharmacol 673: 56-64, 2011.

20. Morley KL, Ferguson PJ and Koropatnick J: Tangeretin and nobiletin induce G1 cell cycle arrest but not apoptosis in human breast and colon cancer cells. Cancer Lett 251: 168-178, 2007.

21. Meiyanto E, Hermawan A and Anindyajati: Natural products for cancer-targeted therapy: Citrus flavonoids as potent chemopreventive agents. Asian Pac J Cancer Prev 13: 427-436, 2012.

22. Vanhoecke BW, Delporte F, Van Braeckel E, Heyerick A, Depypere HT, Nuytinck M, De Keukeleire D and Bracke ME: A safety study of oral tangeretin and xanthohumol administration to laboratory mice. In Vivo 19: 103-107, 2005.

23. Jiang LH, Yang NY, Yuan XL, Zou YJ, Jiang ZQ, Zhao FM, Chen JP, Wang MY and Lu DX: Microarray analysis of mRNA and microRNA expression profile reveals the role of $\beta$-sitosterol-D-glucoside in the proliferation of neural stem cell. Evid Based Complement Alternat Med 2013: 360302, 2013.

24. Chen QL, Lu YY, Zhang GB, Song YN, Zhou QM, Zhang H, Zhang W, Tang XS and Su SB: Characteristic analysis from excessive to deficient syndromes in hepatocarcinoma underlying miRNA array data. Evid Based Complement Alternat Med 2013: 324636, 2013.

25. Sahlgren C, Gustafsson MV, Jin S, Poellinger L and Lendahl U: Notch signaling mediates hypoxia-induced tumor cell migration and invasion. Proc Natl Acad Sci USA 105: 6392-6397, 2008.

26. Eliasz S, Liang S, Chen Y, De Marco MA, Machek O, Skucha S, Miele L and Bocchetta M: Notch-1 stimulates survival of lung adenocarcinoma cells during hypoxia by activating the IGF-1R pathway. Oncogene 29: 2488-2498, 2010.

27. Capaccione KM and Pine SR: The Notch signaling pathway as a mediator of tumor survival. Carcinogenesis 34: 1420-1430, 2013.

28. Cui FB, Li RT, Liu Q, Wu PY, Hu WJ, Yue GF, Ding H, Yu LX, Qian XP and Liu BR: Enhancement of radiotherapy efficacy by docetaxel-loaded gelatinase-stimuli PEG-Pep-PCL nanoparticles in gastric cancer. Cancer Lett 346: 53-62, 2014. 
29. Lin J, Liu C, Gao F, Mitchel RE, Zhao L, Yang Y, Lei J and Cai J: miR-200c enhances radiosensitivity of human breast cancer cells. J Cell Biochem 114: 606-615, 2013.

30. Kurowska EM and Manthey JA: Hypolipidemic effects and absorption of citrus polymethoxylated flavones in hamsters with diet-induced hypercholesterolemia. J Agric Food Chem 52: 2879-2886, 2004.

31. Manthey JA, Cesar TB, Jackson E and Mertens-Talcott S: Pharmacokinetic study of nobiletin and tangeretin in rat serum by high-performance liquid chromatography-electrospray ionization-mass spectrometry. J Agric Food Chem 59: 145-151, 2011.

32. Theys J, Jutten B, Habets R, Paesmans K, Groot AJ, Lambin P, Wouters BG, Lammering $\mathrm{G}$ and Vooijs M: E-Cadherin loss associated with EMT promotes radioresistance in human tumor cells Radiother Oncol 99: 392-397, 2011.

33. Peng Z, Wang CX, Fang EH, Wang GB and Tong Q: Role of epithelial-mesenchymal transition in gastric cancer initiation and progression. World J Gastroenterol 20: 5403-5410, 2014.

34. Vermeulen S, Van Marck V, Van Hoorde L, Van Roy F, Bracke M and Mareel M: Regulation of the invasion suppressor function of the cadherin/catenin complex. Pathol Res Pract 192: 694-707, 1996.

35. Martínez Conesa C, Vicente Ortega V, Yáñez Gascón MJ, Alcaraz Baños M, Canteras Jordana M, Benavente-García O and Castillo J: Treatment of metastatic melanoma B16F10 by the flavonoids tangeretin, rutin, and diosmin. J Agric Food Chem 53: 6791-6797, 2005.
36. Zavadil J, Cermak L, Soto-Nieves N and Böttinger EP: Integration of TGF-beta/Smad and Jagged1/Notch signalling in epithelial-tomesenchymal transition. EMBO J 23: 1155-1165, 2004.

37. Timmerman LA, Grego-Bessa J, Raya A, Bertrán E, PérezPomares JM, Díez J, Aranda S, Palomo S, McCormick F, Izpisúa-Belmonte JC, et al: Notch promotes epithelial-mesenchymal transition during cardiac development and oncogenic transformation. Genes Dev 18: 99-115, 2004.

38. Du X, Cheng Z, Wang YH, Guo ZH, Zhang SQ, Hu JK and Zhou ZG: Role of Notch signaling pathway in gastric cancer: A meta-analysis of the literature. World J Gastroenterol 20: 9191-9199, 2014.

39. Hur K, Toiyama Y, Takahashi M, Balaguer F, Nagasaka T, Koike J, Hemmi H, Koi M, Boland CR and Goel A: MicroRNA-200c modulates epithelial-to-mesenchymal transition (EMT) in human colorectal cancer metastasis. Gut 62: 1315-1326, 2013

40. Zhang CZ, Han L, Zhang AL, Fu YC, Yue X, Wang GX, Jia ZF, Pu PY, Zhang QY and Kang CS: MicroRNA-221 and microRNA-222 regulate gastric carcinoma cell proliferation and radioresistance by targeting PTEN. BMC Cancer 10: 367, 2010.

41. Gattolliat CH, Thomas L, Ciafrè SA, Meurice G, Le Teuff G, Job B, Richon C, Combaret V, Dessen P, Valteau-Couanet D, et al: Expression of miR-487b and miR-410 encoded by $14 \mathrm{q} 32.31$ locus is a prognostic marker in neuroblastoma. Br J Cancer 105: 1352-1361, 2011.

42. Shen J, Niu W, Zhou M, Zhang H, Ma J, Wang L and Zhang H: MicroRNA-410 suppresses migration and invasion by targeting MDM2 in gastric cancer. PLoS One 9: e104510, 2014 